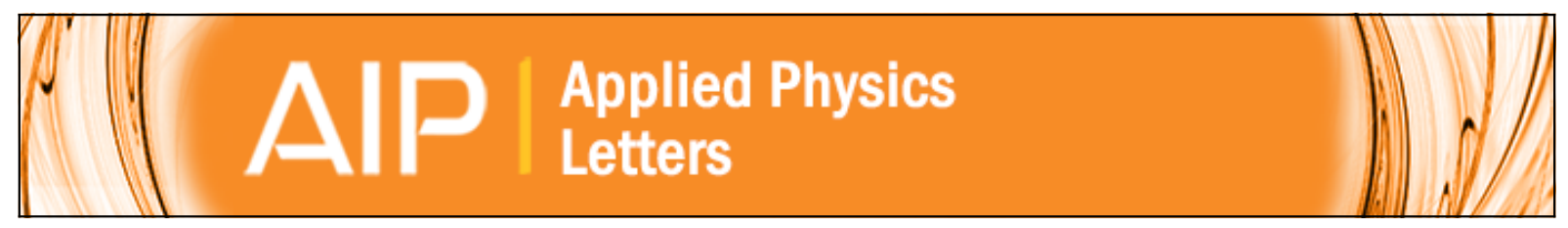

Universal three-way few-electron switch using silicon single-electron transistors Jin He, Zahid A. K. Durrani, and Haroon Ahmed

Citation: Applied Physics Letters 85, 308 (2004); doi: 10.1063/1.1772526

View online: http://dx.doi.org/10.1063/1.1772526

View Table of Contents: http://scitation.aip.org/content/aip/journal/apl/85/2?ver=pdfcov

Published by the AIP Publishing

Over 700 papers \&

presentations on

multiphysics simulation vew now

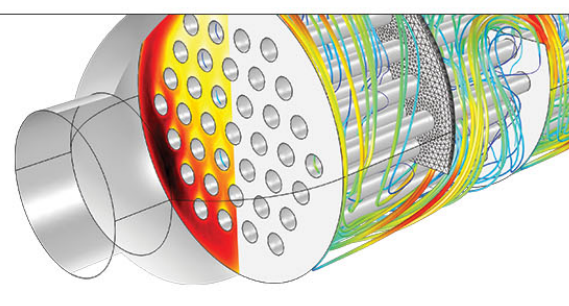




\title{
Universal three-way few-electron switch using silicon single-electron transistors
}

\author{
Jin He, Zahid A. K. Durrani, a) and Haroon Ahmed \\ Microelectronics Research Centre, Cavendish Laboratory, University of Cambridge, Madingley Road, \\ Cambridge CB3 OHE, United Kingdom
}

(Received 4 March 2004; accepted 19 May 2004)

\begin{abstract}
A three-way few-electron switch is implemented using bidirectional electron pumps in silicon-on-insulator material. The switch consists of three branches defined by single-electron transistors, connected to a central node. Any combination of two single-electron transistors forms a bidirectional electron pump. At $4.2 \mathrm{~K}$, each cycle of an rf signal applied to the central node pumps electron packets approximately ten electrons in size through the circuit. It is possible to switch the electron packets in any direction through the branches. The switch may be used for the precise transfer of electrons, and as the basic element in few-electron logic applications. (C) 2004 American Institute of Physics. [DOI: 10.1063/1.1772526]
\end{abstract}

Recent improvements in large-scale integrated (LSI) circuit fabrication techniques have lead to a reduction in the size of electronic devices into the nanometer scale, accompanied by large increases in the circuit operating speed and device density. Present LSI circuits using complementary metal-oxide-semiconductor (CMOS) technology require thousands of electrons to define each "bit". With further miniaturization of electronic devices, statistical fluctuations in the number of electrons become increasingly significant and, ultimately, prevent clear definition of the bits. Singleelectron transistors (SETs), where the Coulomb blockade effect controls electron transport with one-electron precision, ${ }^{1}$ can be used to fabricate circuits where the number of electrons/bits is $\sim 10$ or less. Silicon SETs are of particular interest due to their nanometer scale, low-power dissipation, compatibility with CMOS technology, and the availability of a stable oxide to passivate surface states. These devices have the potential to form the basis of very low-power and highly scalable LSI circuits. ${ }^{2}$

While there have been a number of investigations of single- and few-electron memory devices in silicon, ${ }^{3-5}$ there is comparatively little work on SET logic circuits in silicon. Simple SET logic gates analogous to CMOS designs have now been fabricated. ${ }^{6-8}$ However, it may be difficult to develop complex circuits using these designs due to the low voltage gain in the SET. An alternative approach uses "binary decision diagram" (BDD) switching logic, which does not require voltage gain. ${ }^{9}$ A BDD logic circuit uses two-way switches to guide electron packets through a circuit "tree" representing the logic function, into one of two exit "branches" representing the " 1 " or " 0 " outcome. A two-way switch based on few-electron turnstile operation at $4.2 \mathrm{~K}$, and a BDD AND gate at $1.8 \mathrm{~K}^{11}$ have been demonstrated using $\partial$-doped GaAs SETs.

In this letter, we report the operation at $4.2 \mathrm{~K}$ of a universal three-way electron switch in silicon, where fewelectron packets can be switched in any direction between three different branches connected to a central node. The circuit uses bidirectional electron pumps formed by silicon nanowire SETs, ${ }^{12}$ which transfer electron packets through the

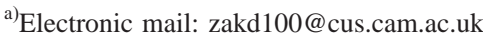

circuit using an rf signal. Electron packets approximately ten electrons, and even as small as two electrons, can be switched by three control gates. The circuit may be used as the basic switching element in BDD logic, and to transfer electrons precisely in complex single-electron logic and memory circuits.

Our switch uses three silicon nanowire SETs ${ }^{13,14}$ (Fig. 1 ), fabricated in the top silicon layer (40 nm thick, doped $n$-type at $2 \times 10^{19} \mathrm{~cm}^{-3}$ with phosphorus) of silicon-oninsulator material. The buried oxide under this layer was $400 \mathrm{~nm}$ thick. Each SET consists of a $50 \mathrm{~nm} \times 100 \mathrm{~nm}$ nanowire, which behaves as a multiple tunnel junction at low temperature. Two side gates control the nanowire current. The circuit was fabricated using a $30 \mathrm{~nm}$ thick Al etch mask,

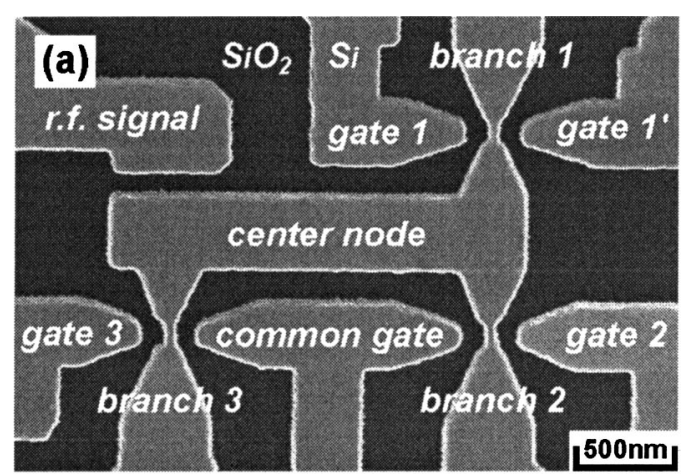

(b)
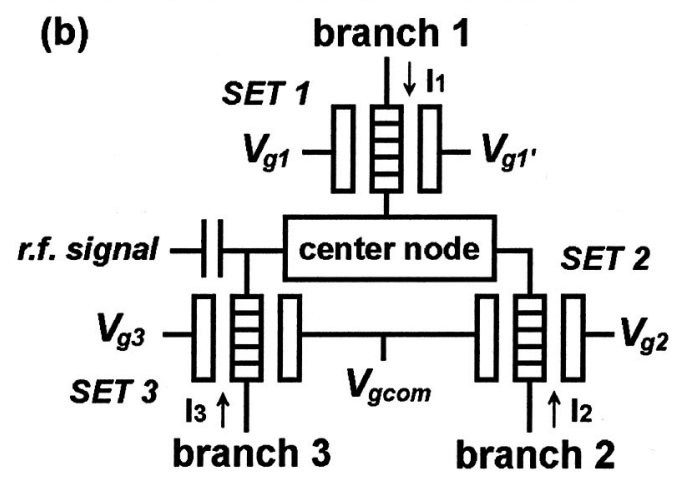

FIG. 1. (a) Scanning electron micrograph of a universal electron switch in silicon-on-insulator material, before oxidation. (b) Circuit diagram of the switch. 

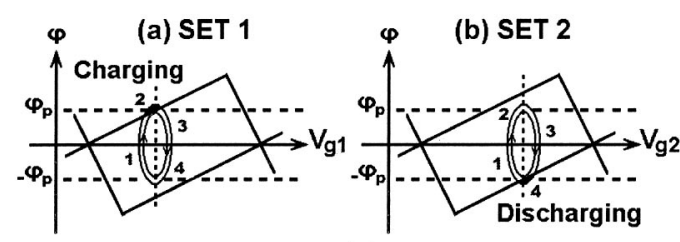

(c)

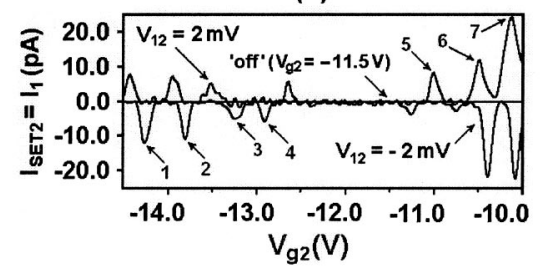

(d)

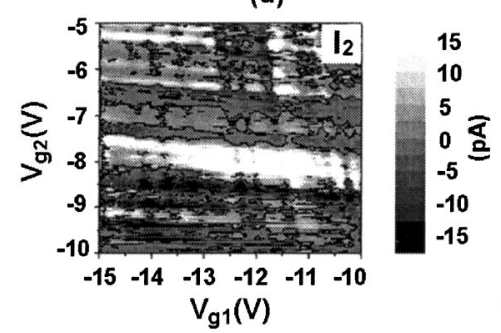

FIG. 2. (a) Schematic of the electron stability diagram of SET1. (b) Schematic of the electron stability diagram of SET2. (c) Single-electron oscillations in SET2 in the $I_{\mathrm{SET} 2}-V_{g 2}$ characteristics. (d) Current in branch $2\left(I_{2}\right)$ at $4.2 \mathrm{~K}$ versus $V_{g 1}$ and $V_{g 2}$. Here $V_{g 3}=-12.5 \mathrm{~V}$.

defined by electron-beam lithography and lift off of the excess metal. The device pattern was then transferred into the silicon by reactive-ion etching in $\mathrm{SiCl}_{4}$ plasma. Next, the $\mathrm{Al}$ was removed by wet etching. Finally, the nanowire cross section was reduced and the surfaces passivated by thermal oxidation for $45 \mathrm{~min}$ at $1000{ }^{\circ} \mathrm{C}$. Figure 1(a) shows a scanning-electron micrograph of the device before oxidation. The width of the silicon region in the nanowire is reduced from $\sim 50 \mathrm{~nm}$ to $\sim 20 \mathrm{~nm}$ after oxidation.

The three SETs form the three branches of the switch, "branch 1", "branch 2", and "branch 3" [Fig. 1(b)]. Currents $I_{1}, I_{2}$, and $I_{3}$ flow in these branches, respectively, with positive polarity when current enters a branch. The branches are connected at a "center node" and any series combination of two SETs forms a bidirectional electron pump. In our switch, there are three electron pumps, pump "A" (SET1 and SET2), pump "B" (SET1 and SET 3) and pump "C" (SET2 and SET3). An rf signal coupled capacitively to the center node drives the pumps. The common gate is not used in the present investigation.

The operation of the bidirectional electron pumps may be understood with reference to pump A. An rf signal is applied to the center node and branch 1 and branch 2 are grounded. The electron number stability diagrams of the SETs are shown schematically in Figs. 2(a) and 2(b), as a function of the gate voltages $V_{g 1}$ and $V_{g 2}$ the center node potential " $\varphi$ ". The trapezoidal regions correspond to areas of Coulomb blockade. As $\varphi$ follows a cycle of the rf signal, the SETs move in and out of Coulomb blockade successively. Along the numbered loop of a cycle, the center node charges at 2 and discharges at 4 . Consequently a packet of electrons is pumped from the terminal of branch 1 to the terminal of branch 2 in each cycle. At another set of gate voltages, electrons may be pumped from the terminal of branch 2 to that of branch 1, implying bidirectionality of the circuit.
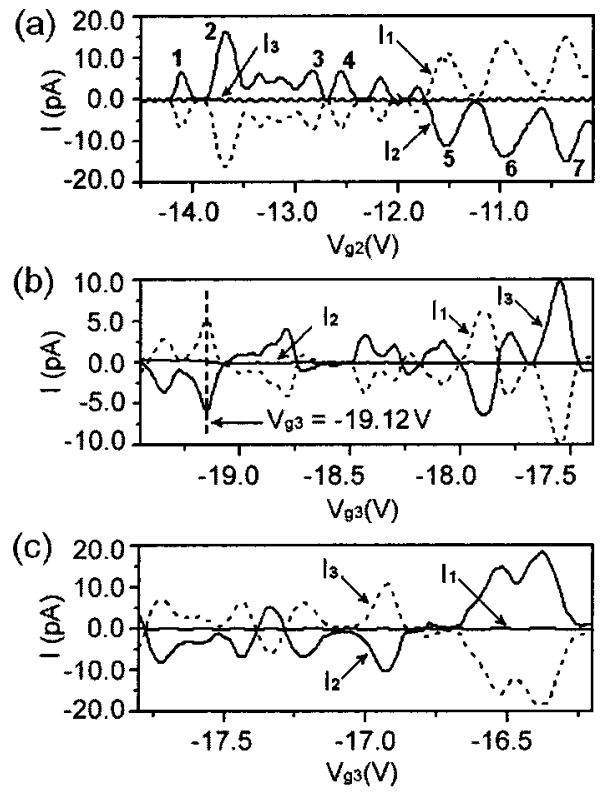

FIG. 3. (a) Current switching between branch 1 and branch 2 at $4.2 \mathrm{~K}$ versus $V_{g 2}$ when $V_{g 1}=-17 \mathrm{~V}$ and $V_{g 3}=-18.9 \mathrm{~V}$. (b) Current switching between branch 1 and branch 3 at $4.2 \mathrm{~K}$ versus $V_{g 3}$ when $V_{g 1}=-9 \mathrm{~V}$ and $V_{g 2}=-11.5 \mathrm{~V}$. (c) Current switching between branch 2 and branch 3 at $4.2 \mathrm{~K}$ versus $V_{g 3}$ when $V_{g 1}=-13 \mathrm{~V}$ and $V_{g 2}=-8 \mathrm{~V}$.

Figure 2(c) shows the Coulomb oscillations of SET2 with SET1 biased in a high conductance regime at $V_{g 1}$ $=0 \mathrm{~V}$ and SET3 open circuited. A voltage $V_{12}$ is applied between branch 1 and branch 2, and Coulomb oscillations are observed w.r.t. $V_{g 2}$ in the SET2 current $I_{\text {SET2 }}=I_{1}$. The characteristics for $V_{12}= \pm 2 \mathrm{mV}$ show asymmetry in the peak positions due to a significant contribution of the gate capacitance to the total charging island capacitance in our SETs. ${ }^{1}$ Our oscillations also do not have an exact period, due to the random nature of island formation in our nanowires. For application in practical circuits, SETs with controlled island size and more periodic oscillations may be necessary, e.g., defined by using a pattern-dependent oxidation method. ${ }^{7}$

Figure 2(d) shows the pumped current $I_{2}$ in grey-scale versus $V_{g 1}$ and $V_{g 2}$ at $4.2 \mathrm{~K}$. Here, the three branches are grounded, $V_{g 3}=-12.5 \mathrm{~V}$ and a $3 \mathrm{MHz}$ and $200 \mathrm{mV}$ peak-topeak sine wave signal is applied to drive the circuit. $V_{g 1}$ and $V_{g 2}$ are varied from $-10 \mathrm{~V}$ to $-15 \mathrm{~V}$ and $-5 \mathrm{~V}$ to $-10 \mathrm{~V}$, respectively. $I_{2}$ oscillates in magnitude if either gate voltage is varied, depending on the operating point of the SETs relative to their electron number stability regions. Both positive (light areas) and negative (dark areas) of current are seen, demonstrating bidirectional electron transfer through the circuit. We observe similar behavior for the currents $I_{1}$ and $I_{3}$ in the other two branches, and the sum of the branch currents at a given combination of $V_{g 1}, V_{g 2}$, and $V_{g 3}$ is zero.

Few-electron packets can be transferred through any of the three ways, in any direction, in our switch by using the control gate voltages $V_{g 1}, V_{g 2}$, and $V_{g 3}$ to adjust the Coulomb gap in the SETs for the selection of the required electron pumps. The remaining gates $\left(V_{g 1^{\prime}}\right.$ and $\left.V_{g \mathrm{com}}\right)$ are not essential for switch operation. Figures 3(a)-3(c) show the transfer of electron packets through the switch using a $3 \mathrm{MHz}$ and $200 \mathrm{mV}$ peak-to-peak rf signal, along the routes formed by branch 1 and branch 2, branch 1 and branch 3, and branch 2 and branch 3 , respectively. In Fig. 3(a), the currents $I_{1}, I_{2}$, and $I_{3}$ are plotted as $V_{g 2}$ and varied from $-10 \mathrm{~V}$ to $-14.5 \mathrm{~V}$ 
$\left(V_{g 1}=-17 \mathrm{~V}\right.$ and $V_{g 3}=-18.9 \mathrm{~V}$ are constant $)$. Here, SET1 and SET2 are "on" and SET3 is "off." We observe that $I_{1}$ $\approx-I_{2}$, and $I_{3} \approx 0 \mathrm{pA}$. When $I_{1}$ is positive, packets of electrons (negative charge) are transferred from branch 2 to branch 1. Conversely, when $I_{1}$ is negative, electrons are transferred from branch 1 to branch 2, demonstrating bidirectional operation. Figure 3(b) shows electron transfer along branch 1 and branch $3\left[I_{1} \approx-I_{3}, I_{2} \approx 0 \mathrm{pA}\right.$, SET1 and SET3 are on, and SET2 is off at $V_{g 2}=-11.5 \mathrm{~V}$, see Fig. 2(c)] and Fig. 3(c) shows electron transfer along branch 2 and branch 3 $\left(I_{2} \approx-I_{3}, I_{1} \approx 0 \mathrm{pA}\right.$, SET2 and SET3 are on, and SET1 is off).

We now compare the pump currents in Fig. 3(a) to the Coulomb oscillations in SET2 [Fig. 2(c)]. In the measurement of Fig. 3(a), SET3 has a maximum Coulomb gap $\sim 30 \mathrm{mV}$, and is off during the entire rf cycle, while SET1 is on with a narrow Coulomb gap at least during part of the rf cycle. In Fig. 2(c), for negative $V_{12}$ (i.e., negative center node voltage relative to branch 2), there are four negative current peaks at points $1-4$. Such a nanowire bias corresponds to the negative part of the rf cycle for the measurement of Fig. 3(a). At these points, SET2 has a high conductance and is likely to be more conductive than SET1, leading to electron transfer from the center node to branch 2. During the positive part of the rf cycle, the asymmetry w.r.t. $V_{12}$ of the Coulomb oscillations in SET2 cause SET1 to conduct better than SET2 and electrons transfer from branch 1 to the center node. This results in four positive peaks in $I_{2}$ in Fig. 3(a) (Nos. 1-4). Conversely, the large current peaks 5-7 in SET2 in Fig. 2(c) for positive $V_{12}$ may lead to the negative current peaks 5-7 in Fig. 3(a) although the situation is very weak for peak No. 6 as there is a large peak for negative $V_{12}$ at $V_{g 2}=-10.4 \mathrm{~V}$. We note that there are small shifts in the position of the peaks w.r.t. $V_{g 2}$ between Figs. 2(c) and 3(a). It is possible that this may be due to variations in the biasing points of the SET when the entire circuit is under bias.

In Fig. 3(b) at $V_{g 3}=-19.12 \mathrm{~V}$, we observe $I_{1}=5.00 \mathrm{pA}$, $I_{2}=0.13 \mathrm{pA}$, and $I_{3}=-5.60 \mathrm{pA}$. Here, $I_{1}+I_{2}+I_{3}=-0.47 \mathrm{pA}$ is the approximate measurement noise level. From the electron pump equation ${ }^{15} I=$ nef, where " $n$ " is the number of electrons and " $f$ " is the pumping frequency, we determine that a current of $5 \mathrm{pA}$ corresponds to approximately ten electrons pumped per rf cycle. We note that the smallest current peaks in Fig. 3 are $\sim 1$ pA, which corresponds to only two electrons pumped per if cycle.

Figure 4 shows the effect of the rf signal frequency and voltage on the current $I_{1}$ in branch 1 as $V_{g 1}$ varies from $-16.7 \mathrm{~V}$ to $-18.9 \mathrm{~V}$. In Fig. 4(a), as the frequency increases, the current peaks increase in magnitude. The current varies approximately linearly with frequency [inset, Fig. 4(a)] and the number of electrons transferred per rf cycle is independent of frequency. In Fig. 4(b), as the amplitude of the rf signal increases, the current peaks increase in magnitude because the SETs operate at larger source-drain voltages. The corresponding electron number per cycle varies approximately linearly with amplitude [inset, Fig. 4(b)]. For small amplitudes, the potential of the center node falls to the extent that all the SETs are in Coulomb blockade and the current falls to zero.

In summary, we have demonstrated a universal threeway electron switch in silicon, where few-electron packets
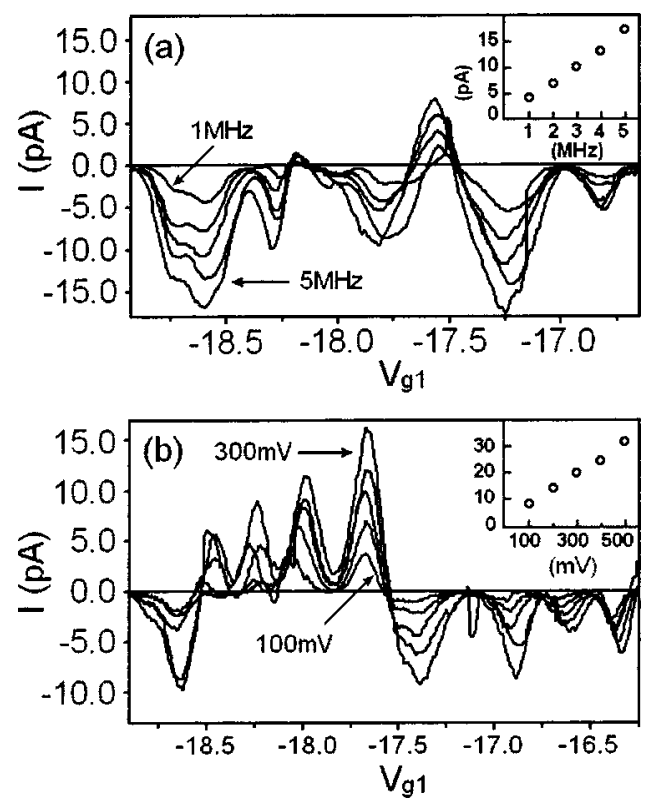

FIG. 4. (a) Frequency dependence of $I_{1}$ versus $V_{g 1}$. The rf signal frequency is varied from $1 \mathrm{MHz}$ to $5 \mathrm{MHz}$ in $1 \mathrm{MHz}$ steps. Here, $V_{g 2}=-12.5 \mathrm{~V}$ and $V_{g 3}=-15.9 \mathrm{~V}$. The inset shows the magnitude of $I_{1}$ at $V_{g 1}=-18.62$ as a function of the frequency. (b) Amplitude dependence of $I_{1}$ versus $V_{g 1}$. The peak-to-peak amplitude of the rf signal is varied from $100 \mathrm{mV}$ to $300 \mathrm{mV}$ in $50 \mathrm{mV}$ steps. The inset shows the number of electrons transferred per rf signal cycle at $V_{g 1}=-17.67 \mathrm{~V}$ as a function of the rf signal amplitude.

can be switched in any direction between three different branches. The switch uses bidirectional electron pumps based on silicon nanowire SETs. At $4.2 \mathrm{~K}$, electron packets approximately ten electrons in size can be transferred. The circuit may be used for precise transfer of electrons, and as the basic element in few-electron logic circuits.

The authors acknowledge many useful discussions with T. Altebaeumer at IBM Zurich, Switzerland. The work was supported by a U. K. EPSRC research grant.

${ }^{1}$ Single Charge Tunneling, edited by H. Grabert and M. H. Devoret (Plenum, New York, 1992).

${ }^{2}$ K. K. Likharev, Proc. IEEE 87, 606 (1999).

${ }^{3}$ K. Yano, T. Ishii, T. Hashimoto, T. Kobayashi, F. Murai, and K. Seki, IEEE Trans. Electron Devices 41, 1628 (1994).

${ }^{4}$ Z. A. K. Durrani, A. C. Irvine, and H. Ahmed, Appl. Phys. Lett. 74, 1293 (1999).

${ }^{5}$ L. Guo, E. Leobandung, and S. Y. Chou, Appl. Phys. Lett. 70, 850 (1997).

${ }^{6}$ C. P. Heij, P. Hadley, and J. E. Mooij, Appl. Phys. Lett. 78, 1140 (2001).

${ }^{7}$ Y. Ono, Y. Takahashi, K. Yamazaki, M. Nagase, H. Namatsu, K. Kurihara, and K. Murase, Appl. Phys. Lett. 76, 3121 (2000).

${ }^{8}$ N. J. Stone and H. Ahmed, Electron. Lett. 35, 1883 (1999).

${ }^{9}$ N. Asahi, M. Akazawa, and Y. Amemiya, IEEE Trans. Electron Devices 44, 1109 (1997).

${ }^{10}$ K. Tsukagoshi and K. Nakazato, Appl. Phys. Lett. 72, 1084 (1998).

${ }^{11}$ K. Tsukagoshi, B. W. Alphenaar, and K. Nakazato, Appl. Phys. Lett. 73, 2515 (1998).

${ }^{12}$ T. Altebaeumer, S. Amakawa, and H. Ahmed, Appl. Phys. Lett. 79, 533 (2001).

${ }^{13}$ R. A. Smith and H. Ahmed, J. Appl. Phys. 81, 2699 (1997).

${ }^{14}$ A. Tilke, R. H. Blick, H. Lorenz, and J. P. Kotthaus, J. Appl. Phys. 89, 8159 (2001).

${ }^{15}$ L. J. Geerligs, V. F. Anderegg, P. A. M. Holweg, J. E. Mooij, H. Pothier, D. Esteve, C. Urbina, and M. H. Devoret, Phys. Rev. Lett. 64, 2691 (1990). 